\title{
Axial parkinsonian symptoms can be improved: the role of levodopa and bilateral subthalamic stimulation
}

Boulos-Paul Bejjani, David Gervais, Isabelle Arnulf, Savas Papadopoulos, Sophie Demeret, Anne-Marie Bonnet, Philippe Cornu, Philippe Damier, Yves Agid

\begin{abstract}
Objective-To assess the effects of high frequency stimulation of the subthalamic nucleus (STN) on axial symptoms occurring in advanced stages of Parkinson's disease (PD).
\end{abstract}

Methods-The efficacy of STN stimulation on total motor disability score (unified Parkinson's disease rating scale (UPDRS) part III) were evaluated in 10 patients with severe Parkinson's disease. The subscores were then studied separately for limb akinesia, rigidity, and tremor, which are known to respond to levodopa, and axial signs, including speech, neck rigidity, rising from a chair, posture, gait, and postural stability, which are known to respond less well to levodopa. Patients were clinically assessed in the "off" and "on" drug condition during a levodopa challenge test performed before surgical implantation of stimulation electrodes and repeated 6 months after surgery under continuous STN stimulation. A complementary score for axial symptoms from the "activities of daily living" (ADL) - that is, speech, swallowing, turning in bed, falling, walking, and freezing-was obtained from each patient's questionnaire (UPDRS, part II).

Results-Improvements in total motor disability score $(62 \%)$, limb signs $(62 \%)$, and axial signs (72\%) obtained with STN stimulation were statistically comparable with those obtained with levodopa during the preoperative challenge $(68 \%, 69 \%$, and $59 \%$, respectively). When levodopa and STN stimulation were combined there was a further improvement in total motor dis- ability (80\%) compared with preoperative levodopa administration. This consisted largely of an additional improvement in axial signs (84\%) mainly for posture and postural stability, no further improvement in levodopa responsive signs being found. Axial symptoms from the ADL showed similar additional improvement when levodopa and STN stimulation were combined.

Conclusion-These findings suggest that bilateral STN stimulation improves most axial features of Parkinson's disease and that a synergistic effect can be obtained when stimulation is used in conjunction with levodopa treatment.

(F Neurol Neurosurg Psychiatry 2000;68:595-600)

Keywords: Parkinson's disease; subthalamic nucleus; deep brain stimulation; axial symptoms; levodopa

Patients with Parkinson's disease tend to respond less well to long term levodopa treatment after several years of evolution of the disease. ${ }^{1}$ However, limb akinesia, rigidity, and tremor continue to be markedly improved by levodopa treatment, even in late stages of the disease. $^{2}$ The overall decrease in the effectiveness of levodopa during the course of the disease has been attributed to the appearance of axial symptoms, such as dysarthria, gait disorders, and postural instability, which in time largely contribute to the motor handicap of patients and respond poorly to levodopa. ${ }^{23}$ The progressive worsening of axial features in Parkinson's disease is considered to result from the increasing severity of non-dopaminergic lesions affecting brain areas localised outside

Neurochirurgie, Groupe Hospitalier Pitié-Salpêtrière,

Paris, France

P Cornu

Departement de Neurologie, Hôpital Hôtel-Dieu de France, USJ, Beirut, Lebanon B-P Bejiani

Correspondence to: Dr Yves Agid, Centre d'Investigation Clinique, Groupe Hospitalier Pitié-Salpêtrière, 47 boulevard de l'Hôpital 75013 Paris, France cic.salpetriere@, psl.ap-hop-paris.fr

\begin{tabular}{|c|c|c|c|c|c|c|c|}
\hline \multirow[b]{2}{*}{ Patients } & \multirow[b]{2}{*}{ Age (y) } & \multirow[b]{2}{*}{ Sex } & \multirow{2}{*}{$\begin{array}{l}\text { Disease } \\
\text { duration } \\
(y)\end{array}$} & \multicolumn{2}{|c|}{ Hoehn and Yahr score "offlon" } & \multicolumn{2}{|c|}{$\begin{array}{l}\text { Dosage of levodopa } \\
\text { equivalents ( } m g / \text { day) }\end{array}$} \\
\hline & & & & Before surgery & After surgery & $\begin{array}{l}\text { Before } \\
\text { surgery }\end{array}$ & $\begin{array}{l}\text { After } \\
\text { surgery }\end{array}$ \\
\hline 1 & 40 & $\mathrm{M}$ & 15 & $5 / 4$ & $1.5 / 1$ & 1250 & 1100 \\
\hline 2 & 45 & $\mathrm{M}$ & 11 & $5 / 4$ & $1 / 0$ & 1550 & 525 \\
\hline 3 & 64 & $\mathrm{~F}$ & 28 & $4 / 3$ & $1.5 / 1$ & 1150 & 300 \\
\hline 4 & 50 & $\mathrm{M}$ & 10 & $3 / 1.5$ & $1 / 1$ & 1550 & 200 \\
\hline 5 & 54 & $\mathrm{M}$ & 17 & $4 / 2$ & $2 / 1$ & 600 & 250 \\
\hline 6 & 65 & M & 11 & $5 / 2.5$ & $2 / 1$ & 1450 & 450 \\
\hline 7 & 74 & $\mathrm{~F}$ & 25 & $3 / 1$ & $1 / 0$ & 350 & 0 \\
\hline 8 & 46 & $\mathrm{~F}$ & 15 & $5 / 3$ & $1.5 / 1$ & 1500 & 500 \\
\hline 9 & 46 & $\mathrm{M}$ & 11 & $3 / 2$ & $1 / 0$ & 1250 & 300 \\
\hline 10 & 61 & $\mathrm{~F}$ & 8 & $4 / 2$ & $2 / 1$ & 1750 & 475 \\
\hline Mean (SEM) & $54(3)$ & & $15(2)$ & $4.1(0.3) / 2.5(0.3)$ & $1.5(0.1 / 0.1)^{\star} 6(0.2)^{\star}$ & $1240(140)$ & $410(90)^{\star}$ \\
\hline
\end{tabular}




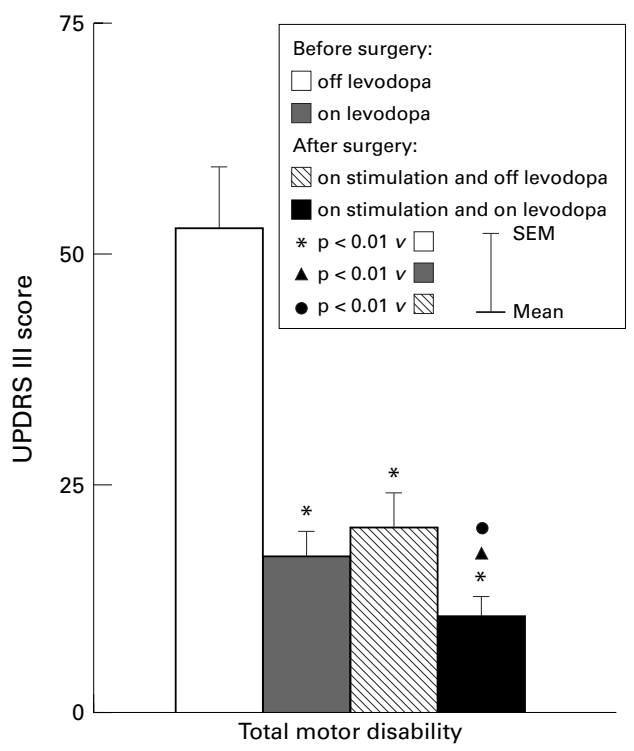

Figure 1 Effects of administration of a single dose of levodopa on total parkinsonian motor disability score (UPDRS part III).

the output pathways downstream from the basal ganglia. ${ }^{4}$ The possibility that decreased dopamine transmission within the basal ganglia contributes to this axial symptomatology, however, cannot be ruled out. ${ }^{5}$

The recent reintroduction of stereotactic neurosurgery in the treatment of patients with advanced Parkinson's disease has provided evidence that lesioning ${ }^{6}$ or chronic stimulation ${ }^{7}$ of the posteroventral medial part of the pallidum improve both parkinsonian symptoms and levodopa induced dyskinesias (LIDs), whereas axial features are not improved except during the first year of treatment. ${ }^{6}$ Our experience in the treatment of patients with Parkinson's disease by continuous bilateral high frequency stimulation of the subthalamic nucleus (STN) suggested that the marked improvement in parkinsonian motor disability was accompanied by a significant improvement in axial symptoms.

\section{Methods}

SUBJECTS

Ten patients with severe idiopathic Parkinson's disease (table) were enrolled in this study 6 months after having been treated by bilateral STN high frequency stimulation. Before surgery, they have a clear response to levodopa (more than 50\% improvement on the unified Parkinson's disease rating scale ${ }^{8}$ (UPDRS) part III during acute levodopa challenge as shown in fig 1) but experienced severe motor fluctuations (4.4 (SEM 0.4), UPDRS part IV B) and disabling LIDs (7.3 (SEM 0.6), UPDRS part IV A). The surgical procedure for electrode implantation was performed using stereotactic MRI $^{10}$ The STN was localised by direct visualisation on three dimensional rendered T2 weighted MR acquisitions, guided by intraoperative electrophysiological recording and exploratory stimulation. The extremity of each implanted electrode (Medtronic, Minneapolis, MN, USA) contained four contacts (numbered 0 to 3, 0 being the most distal) over a distance of $7.5 \mathrm{~mm}$, through which current could be applied separately. The optimal settings for each of the therapeutic electrical variables were determined 10 days after surgery according to the results of the evaluation of the effects of stimulation performed during our standardised protocol previously described. ${ }^{10}$ Six months after surgery, the voltage ranged from 1.8 to 3.3 $\mathrm{V}$, pulse width from 60 to $90 \mu$ s and frequency from 130 to $185 \mathrm{~Hz}$. This current was exclusively monopolar and was applied bilaterally through one contact in five patients $(1,2,4$, 8, and 9), and through one contact on one side and two contacts on the other side in the other five (3, 5-7, and 10). Patients have a marked improvement of their disease with a $76 \%$
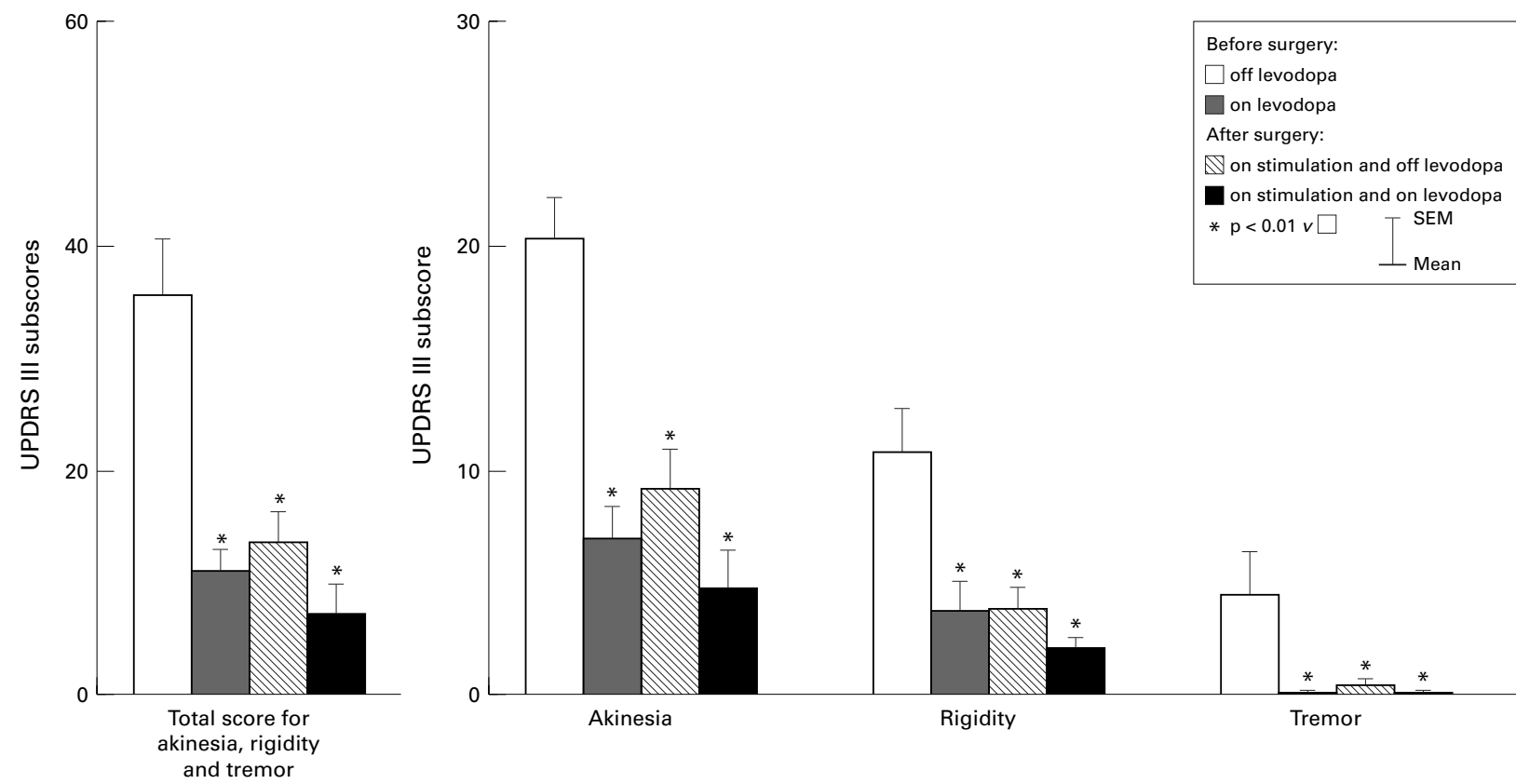

Figure 2 Effects of administration of a single dose of levodopa on total and detailed scores for limb signs (UPDRS part III). 
improvement in the Hoehn and Yahr parkinsonian motor disability score ${ }^{11}$ in the "on" state, and a $62 \%$ reduction in the daily dosage of levodopa, compared with the preoperative state (table). The LIDs and motor fluctuations score (UPDRS part IV A (1.4 (SEM 0.5) and B (0.4 (SEM 0.3)) were decreased by $80 \%$ and $90 \%$, respectively.

\section{ASSESSMENT OF PARKINSONIAN DISABILITY}

Global motor disability was assessed using the UPDRS part III motor score during a levodopa challenge performed in fasting conditions after a 12 hour period of drug withdrawal, using a 50 mg suprathreshold dose of levodopa. The motor score was obtained in the "off" drug state before levodopa administration and in the "on" state 1 hour after levodopa administration, at the time of maximal clinical improvement. This evaluation was performed during the month preceding neurosurgery and was repeated in the same conditions under continuous STN stimulation 6 months after surgery, using an identical levodopa dose and performed by the same investigator.

The scores for limb tremor (items 20 (except face, lips, and chin tremor) and 21), limb rigidity (item 22 (except neck rigidity) and limb akinesia (items 23, 24, 25, and 26)) were assessed separately using the corresponding subscales of the total UPDRS score. The score for axial features was calculated using the subscales of the UPDRS part III score: speech, neck rigidity, rising from a chair, posture, gait, and postural instability (items 18, 22 (neck rigidity only), 27, 28, 29, and 30 of the total UPDRS score). A subjective scoring of axial features was also used and was calculated using the following subscales for the UPDRS part II score: speech, swallowing, turning in bed,
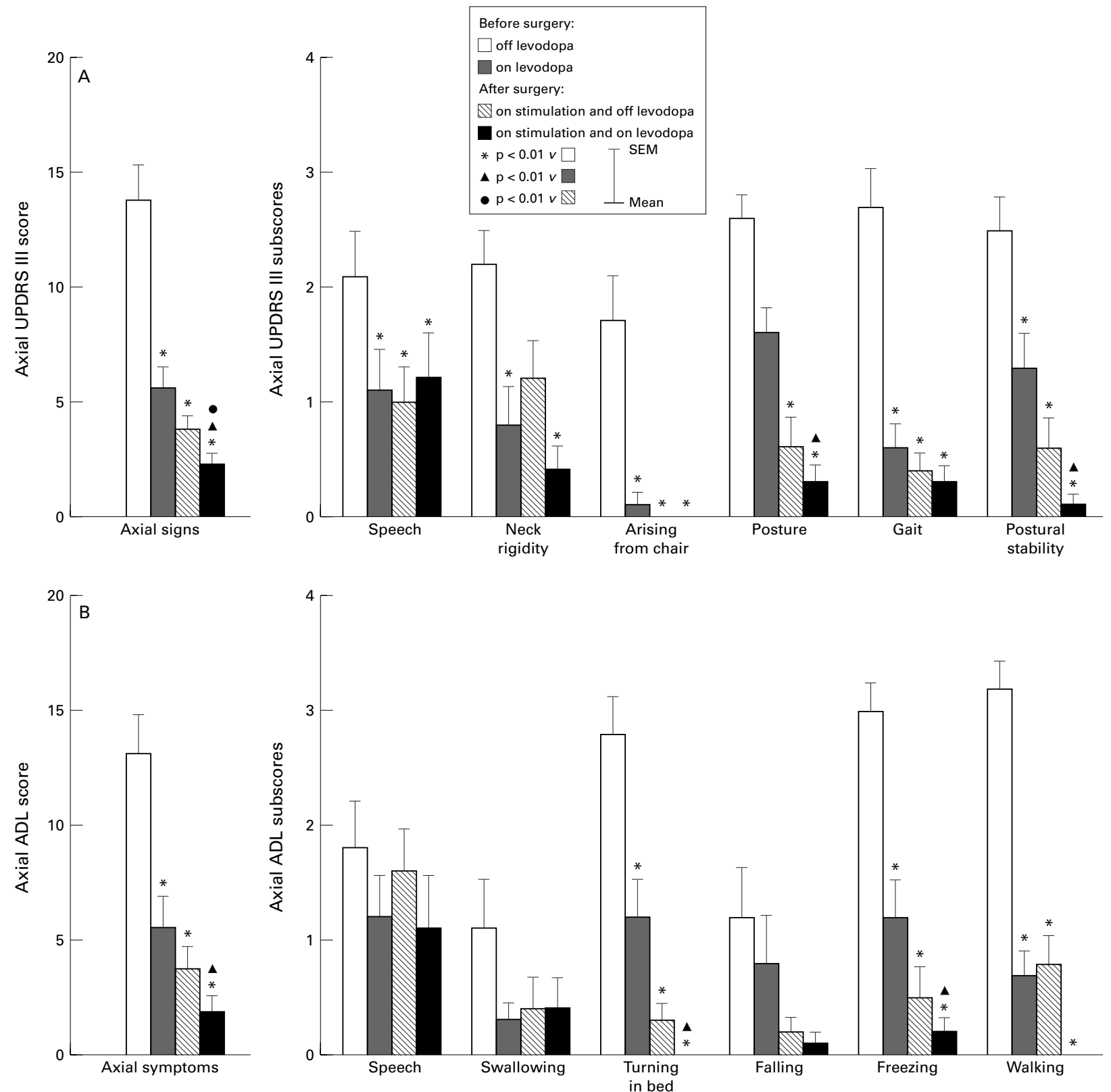

Figure 3 Effects of administration of a single dose of levodopa on total and detailed scores for (A) axial signs (UPDRS part III) and (B) axial symptoms (UPDRS part II). 
falling, freezing, and walking (items $5,7,12$, 13,14 , and 15 of the total UPDRS score).

DATA ANALYSES

Statistical analyses of the data were performed on the patients' scores obtained during the "off" and "on" levodopa states before surgery and after surgery under bilateral STN stimulation. Statistical comparisons of the different motor states were performed using the Wilcoxon signed rank paired statistical test for non-parametric data and a paired Student's $t$ test whenever the variable followed a normal distribution. To avoid type I errors, a $p<0.01$ threshold for significance was chosen for all analyses.

\section{Results}

TOTAL PARKINSONIAN MOTOR DISABILITY

Before surgery, a single dose of levodopa improved the total motor disability score (UPDRS part III score) by $68 \%$. Six months after surgery, this score was improved by $62 \%$ (by comparison with the preoperative score), when the patient was under continuous STN stimulation and without levodopa. The combination of STN stimulation and levodopa administration induced a significantly greater motor improvement $(80 \%)$ than that obtained preoperatively with levodopa $(\mathrm{p}<0.01)$ or postoperatively with STN stimulation alone $(\mathrm{p}<0.01$, fig 1$)$.

\section{LIMB SIGNS}

The administration of a single dose of levodopa improved the scores for limb signs (akinesia, rigidity, and tremor) by $69 \%$. Stimulation of the STN alone or in combination with levodopa treatment did not produce a significant additional improvement, whether the total score for limb signs or the detailed scores (akinesia, rigidity, and tremor) were considered (fig 2).

\section{AXIAL SIGNS}

During the preoperative challenge, levodopa administration improved axial motor signs by $59 \%$ (fig $3 \mathrm{~A}$ ). During the postoperative test there was a $72 \%$ reduction in the score of axial signs after STN stimulation without levodopa. Compared with either levodopa alone $(\mathrm{p}<0.01)$ or STN stimulation alone $(\mathrm{p}<0.01)$, the combination of STN stimulation and levodopa administration provided a further significant improvement in the axial motor disability score $(84 \%)$. When evaluated separately, the subscores for axial signs were heterogeneously modified by levodopa and STN stimulation (fig 3 A). Some scores, such as those for speech disorders and neck rigidity, displayed mild to moderate improvement with levodopa comparable with that achieved with STN stimulation alone or combined with levodopa. Others, such as those for getting up from a chair and gait, were markedly improved by either levodopa or STN stimulation. Abnormal posture and postural stability were only partially improved by levodopa (38\% and $48 \%$ respectively) and showed a greater improvement with STN stimulation ( $77 \%$ and $76 \%$ respectively).
When STN stimulation and levodopa treatment were combined, they resulted in a greater improvement $(88 \%$ and $96 \%(\mathrm{p}=0.04)$ respectively) than levodopa alone.

When axial parkinsonian symptoms, estimated from each patient's questionnaire (activities of daily living, UPDRS part II) were considered (fig $3 \mathrm{~B}$ ), they displayed similar results to those for axial signs on UPDRS part III. Turning in bed and freezing followed a similar pattern of improvement to posture and postural stability, with a greater improvement being achieved through the combination of levodopa and stimulation than with levodopa alone.

\section{Discussion}

In patients responding well to antiparkinsonian drugs, axial features were found to respond partially to levodopa, an effect that is generally close to that obtained under STN stimulation. Interestingly, the effects of levodopa were potentiated by the adjunction of STN stimulation. This additional improvement of axial features (except for speech, swallowing difficulties, and neck rigidity) suggests the existence of a synergistic effect between levodopa and STN stimulation, an effect that was not found for limb signs (akinesia, rigidity, and tremor).

Although small in number, the study population consisted of a group of highly levodopa responsive patients with a homogeneous clinical picture. Furthermore, the results of UPDRS part II should be treated with caution because of the subjective nature of the scoring. However, the high degree of consistency in the results of all patients strongly suggests that the results are reliable.

The fact that the improvement in motor score for limb akinesia, rigidity and tremor, and gait produced by STN stimulation was equivalent to that obtained preoperatively with levodopa administration is consistent with other recent findings. ${ }^{12}{ }^{13}$ It also suggests that the improvement obtained preoperatively for these particular symptoms with levodopa administration is a good predictor of the postoperative improvement to be expected with STN stimulation. The enhanced effects of STN stimulation on axial features when this form of treatment was combined with levodopa administration (mainly accounting for improved posture and postural stability) differ, however, from the results reported by Limousin et $a l^{13}$ even when using the same threshold of significance. This variation could be due to: (1) differences in neurosurgical procedure, since the trajectory used for electrode implantation by our group was more oblique laterally and thus could have covered a greater part of the STN; (2) differences in the volume of the STN stimulated, as we used monopolar stimulation through two of the electrode contacts in five of our patients whereas Limousin et al used a single contact in each patient.

The partial efficacy of levodopa on axial signs confirms previous reports that some axial signs are alleviated by levodopa. In particular, turning in bed was previously found to be restored to normal by levodopa for most 
patients with Parkinson's disease (18 out of 19 patients $\left.s^{5}\right)$. This result is not surprising as akinesia and rigidity, which are considerably improved by levodopa therapy, contribute at least partially to axial scores. The only partial $(57 \%)$ preoperative responsiveness of this symptom in our study (fig $3 \mathrm{~B}$ ) was probably due to the lack of night time levodopa coverage. Postoperatively, continuously (24 hours a day) applied STN stimulation completely suppressed this symptom during the night, thus explaining the apparent synergistic effect between levodopa and stimulation. On the other hand, the synergistic effect obtained in the improvement of freezing might also be explained by the drastic reduction in levodopa intake made possible by STN stimulation, levodopa exposure having been incriminated in "motor blocks". ${ }^{14}$ At least in these levodopa responsive forms of Parkinson's disease, levodopa efficacy on axial signs shows that the central dopaminergic neuronal loss contributes to the severity of axial signs.

After six months' continuous bilateral stimulation of the STN, the therapeutic effect on axial signs did not differ from that of levodopa. This result could mean a "dopamine-like" effect, as if STN stimulation re-established dopaminergic transmission in a similar manner to levodopa. This theory is compatible with the classic anatomical model in which STN "neuroinhibition", ${ }^{15}$ located downstream from the nigrostriatal dopaminergic pathway, reestablishes the normal functioning of the striato-pallido-subthalamo-pallido-thalamocortical output. This mechanism, is however, insufficient to explain the synergistic effects of levodopa combined with STN stimulation on posture and postural stability.

As also reported in previous studies, ${ }^{23}$ axial signs were not completely alleviated by levodopa. This is compatible with the hypothesis that non-dopaminergic lesions are also responsible for axial symptomatology. Any such neuronal loss could not be located downstream from the nigrostriatal dopaminergic pathway output, or levodopa would not have such a profound effect on the cardinal symptoms of Parkinson's disease. We therefore hypothesise that these non-dopaminergic lesions are located in "parallel" to the dopaminergic nigrostriatal pathway ${ }^{4}$ and thus do not impede the beneficial action of levodopa on dopamine responsive symptoms. Non-dopaminergic lesions have been described in Parkinson's disease, ${ }^{16-18}$ located in the cerebral cortex, the basalis nucleus of Meynert, the pedunculopontine nucleus, the locus ceruleus, the dorsal vagus nucleus, and the raphe nuclei, but the role of these lesions in the appearance of axial symptoms remains unknown. Thus, in this regard, the possibility that high frequency stimulation of the STN restores normal functions of non-dopaminergic neuronal circuits can be considered. Although the precise mechanism of stimulation is unknown, the beneficial effect of STN stimulation necessarily involves the nucleus itself and related structures. The STN is a good candidate to explain the effects of stimulation on axial signs as the
STN has been claimed to have a role in the control of postural reactions ${ }^{19}$ but this has yet to be confirmed. Among the connections of the STN that are not directly related to dopaminergic pathways, such as the cerebral cortex, ${ }^{20}$ the parafascicular nucleus of the thalamus, ${ }^{21}$ and the pedunculopontine nucleus, ${ }^{22}$ only the last receives a direct output from the $\mathrm{STN} .^{23}$ Although the precise physiological role of the pedunculopontine nucleus is still unknown, it has been suggested that it is involved in the relay of information for control of postural functions during locomotion. ${ }^{24}$ The fact that it projects bilaterally to basal ganglia structures ${ }^{22}$ increases the likelihood of its being implicated in axial functions. The modulation of this pathway by STN stimulation could thus be a plausible explanation for the synergistic effect between levodopa and STN stimulation, mainly seen on posture and postural stability in our study.

Whatever the mechanism involved, bilateral STN stimulation alleviates all cardinal features of parkinsonism and therefore constitutes a highly efficient treatment of Parkinson's disease. Not only were limb signs (akinesia, rigidity, and tremor) greatly improved, in line with previous reports, ${ }^{12} 13$ but also axial features (turning in bed, falling, freezing, posture, postural stability, gait). This technique would therefore seem to be a highly suitable therapy for axial signs responding partially to levodopa in Parkinson's disease. However, some axial features, such as dysarthria, swallowing impairment, and neck rigidity, were relatively resistant to either levodopa therapy or STN stimulation. Our results are applicable only to the axial disability encountered in patients with idiopathic Parkinson's disease and not to axial predominant parkinsonism or "parkinsonian plus" syndromes, as the last two were exclusion criteria for surgery. These results, obtained in patients presenting severe and levodopa responsive Parkinson's disease, need to be confirmed in other less levodopa responsive clinical forms of the disease.

We are indebted to Drs Didier Dormont and Bernard Pidoux for their contribution, and to the medical staff of the clinical investigation centre for their support.

1 Barbeau A, Roy M. Six-year results of treatment with levodopa plus benserazide in Parkinson's disease. Neurology 1976;26:399-404.

2 Bonnet A-M, Loria Y, Saint-Hilaire $\mathrm{MH}$, et al. Does long-term aggravation of Parkinson's disease result from
nondopaminergic lesions? Neurology 1987;37:1539-42.

3 Klawans HL. Individual manifestations of Parkinson's disease after 10 or more years of levodopa. Mov Disord 1986;1:187-92.

4 Agid Y. Parkinson's disease: pathophysiology. Lancet 1991; 337:1321-4.

5 Steiger MJ, Thompson PD, Marsden CD. Disordered axial movement in Parkinson's disease. F Neurol Neurosurg Psychiatry 1996;61:645-8.

6 Lang AE, Lozano AM, Montgomery E, et al. Posteroventral medial pallidotomy in advanced Parkinson's disease. $N$ Engl F Med 1997;337:1036-42.

7 Pahwa R, Wilkinson S, Smith D, et al. High-frequency stimulation of the globus pallidus for the treatment of Parkinson's disease. Neurology 1997;49:249-53.

8 Fahn S, Elton R. Unified Parkinson's disease rating scale. In: Fahn S, Marsden CD, Calne D, et al, eds. Recent developments in Parkinson's disease. 2nd ed. New Jersey: MacMillan Healthcare Information, 1987:153-63.

9 Dormont D, Cornu P, Pidoux B, et al. Chronic thalamic stimulation with three-dimensional MR stereotactic guidance. Am f Neuroradiol 1997;18:1093-107.

10 Bejiani B, Damier P, Arnulf I, et al. Pallidal stimulation for Bejjani B, Damier P, Arnulf I, et al. Pallidal stimulation for
Parkinson's disease. Two targets? Neurology 1997;49:156469. 
11 Hoehn MM, Yahr MD. Parkinsonism: onset, progression, and mortality. Neurology 1967;17:427-42.

12 Kumar R, Lozano AM, Kim YJ, et al. Double-blind evaluation of subthalamic nucleus deep brain stimulation in advanced Parkinson's disease. Neurology 1998;51:850-5.

13 Limousin P, Krack P, Pollak P, et al. Electrical stimulation of the subthalamic nucleus in advanced Parkinson's disease. N Engl f Med 1998;339:1105-11.

14 Giladi N, McMahon D, Przedborski S, et al. Motor blocks in Parkinson's disease. Neurology 1992;42:333-9.

15 Benabid AL, Pollak P, Gross C, et al. Acute and long-term effects of subthalamic nucleus stimulation in Parkinson's disease. Stereotact Funct Neurosurg 1994;62:76-8.

16 Escourolle R. Anatomic lesions in Parkinson's disease and parkinsonian syndromes. Ann Med Interne (Paris) 1972; 123:349-56.

17 Agid Y, Cervera P, Hirsch E, et al. Biochemistry of Parkinson's disease 28 years later: a critical review. Mov Disord

18 Jellinger K. Overview of morphological changes in Parkinson's disease. Adv Neurol 1987;45:1-18.

19 Martin JP. The basal ganglia and posture. London: Pitman Medical, 1967:1-152.
20 Nambu A, Tokuno $\mathrm{H}$, Inase $\mathrm{M}$, et al. Corticosubthalamic input zones from forelimb representations of the dorsal and ventral divisions of the premotor cortex in the macaque monkey: comparison with the input zones from the primary motor cortex and the supplementary motor area. Neurosci Lett 1997;239:13-16.

21 Féger J, Bevan M, Crossman AR. The projections from the parafascicular thalamic nucleus to the subthalamic nucleus and the striatum arise from separate neuronal populations: a comparison with the corticostriatal and corticosubthaamic efferents in a retrograde fluorescent double-labelling study. Neuroscience 1994;60:125-32.

22 Lavoie B, Parent A. Pedunculopontine nucleus in the squirrel monkey: distribution of cholinergic and monoaminergic neurons in the mesopontine tegmentum with evidence for the presence of glutamate in cholinergic neurons. 7 Comp Neurol 1994;344:190-209.

23 Smith Y, Hazrati LN, Parent A. Efferent projections of the unthe subthalamic nucleus in the squirrel monkey as studied by 1990;294:306-23. 24 Garcia-Rill E. The basal ganglia and the locomotor regions. Brain Res 1986;396:47-63. 